\title{
Effects of Preemptive Analgesia with Parecoxib Sodium on Haemodynamics and Plasma Stress Hormones in Surgical Patients with Thyroid Carcinoma
}

\author{
Lian-Dong Wang ${ }^{1 \&}$, Xia Gao ${ }^{2 \&}$, Jun-Ying Li², Hong-Yan Yu ${ }^{3}$ Hai-Wen $\mathrm{Su}^{3 *}$, \\ Lian-Zhong Liư ${ }^{4}$, Jun Qi ${ }^{4}$
}

\begin{abstract}
Background: To investigate the effects of preemptive analgesia with parecoxib sodium on haemodynamics and plasma stress hormones in surgical patients with thyroid carcinoma. Materials and Methods: Fifty-seven patients with thyroid carcinoma who underwent thyroidectomy selectively in Laiwu Hospital Affiliated to Taishan Medical University and Binzhou People's Hospital were selected and randomly divided into three groups, 19 cases in each group. The control group was intravenously injected $0.9 \%$ sodium chloride injection before anesthesia induction; trial group I was intravenously injected with parecoxib sodium 20 min before anesthesia induction; based on trial group I, trial group II was injected with parecoxib sodium again $12 \mathrm{~h}$ after surgery. The levels of plasma norepinephrine (NE), cortisol (Cor) and blood glucose before, 12 and $24 \mathrm{~h}$ after surgery and changes of haemodynamics before surgery, at the end of surgery and 12, 24 and $48 \mathrm{~h}$ after surgery were compared in the three groups. Besides, visual analogue scale (VAS) scores were recorded 4, 8, 12 and $24 \mathrm{~h}$ after surgery. Results: 12 and $24 \mathrm{~h}$ after surgery, the levels of plasma $\mathrm{NE}$ and Cor in three groups rose dramatically $(P<0.05$ or $(P<0.01)$; The levels of plasma NE and Cor in trial groups I and II were evidently lower than in control group $(P<0.05$ or $P<\mathbf{0 . 0 1}$ ), and those in trial group II slightly lower than in trial group I. $12 \mathrm{~h}$ after surgery, the heart rates (HR) and systolic pressures (SBP) in trial groups I and II increased obviously by comparison to surgery before $(P<0.05$ or $P<0.01)$, but gradually returned to the preoperative level. HR, SBP and diastolic pressures (DBP) in trial groups I and II at the end of surgery and $12 \mathrm{~h}$ after surgery were all lower than in the control group, and significant differences were present $(P<0.05$ or $(P<0.01)$. At $4,8,12$ and $24 \mathrm{~h}$ after surgery, VAS scores in trial groups I and II were markedly lower than in the control group $((P<0.05$ or $(P<0.01)$, the scores in trial group II being the lowest. Conclusions: Combined application of parecoxib sodium for preemptive analgesia before anesthesia and after surgery can effectively reduce the levels of plasma stress hormones and improve analgesic effects in surgical patients with thyroid carcinoma, and without conspicuous impact on haemodynamics.
\end{abstract}

Keywords: Thyroid carcinoma - parecoxib sodium - preemptive analgesia - haemodynamics - stress hormones

Asian Pac J Cancer Prev, 16 (9), 3977-3980

\section{Introduction}

Thyroid carcinoma, the most common malignant tumors in the thyroid, originates from thyroid epithelial cells. In recent years, the incidence of thyroid carcinoma is on the rise. It has become one of the malignant solid tumors with the fast growth rate in almost two decades (Cramer et al., 2010). At current, comprehensive treatment dominated by surgery is a major way to treat thyroid carcinoma. Positive and proper surgery can not only prolong the survival of patients with thyroid carcinoma, but also decrease the recurrence rate and ameliorate quality of life. Nevertheless, with thyroid surgery increasing continuously, noxious stimulation induced by surgical incision and postoperative pain can make peripheral and central nervous systems hypersensitive so as to cause stress responses, which is unfavorable to postoperative prognosis (Persec et al., 2007).

As a dominating link in various modes of analgesia, preemptive analgesia can alleviate stress responses in perioperative period by protecting peripheral and central nervous systems from sensitization, which is of great importance in the improvement of postoperative prognosis (Sandhu et al., 2011). Parecoxib sodium, a water-soluble prodrug of valdecoxib, can reduce the synthesis of peripheral prostaglandin to exert the effect of analgesia and relieving inflammation and via inhibition of peripheral epoxidase-2 (COX-2) expression. Additionally, it can also

${ }^{I}$ Department of General Surgery, Laiwu Hospital Affiliated to Taishan Medical University, Laiwu, ${ }^{2}$ Parmacy Department, Binzhou People's Hospital, Binzhou, ${ }^{3}$ Department of Anesthesiology, The Third People's Hospital of Qingdao, Qingdao, ${ }^{4}$ Department of Anesthesiology, Laiwu Hospital Affiliated to Taishan Medical University, Laiwu, China ${ }^{\circledR}$ Equal contributors *For correspondence: suhaiwen999@126.com 
prevent central sensitization through inhibition of central COX-2 expression (Wei et al., 2013). In this study, the changes of plasma stress hormones and haemodynamics in surgical patients with thyroid carcinoma before and after application of parecoxib sodium were observed in order to investigate its impact on stress responses and haemodynamics in perioperative period.

\section{Materials and Methods}

\section{General data}

Fifty-seven patients with thyroid carcinoma who underwent thyroidectomy selectively in Laiwu Hospital Affiliated to Taishan Medical University and Binzhou People's Hospital from Jan., 2012 to Jan., 2015 were selected, in which the males and females were 23 and 34 cases, respectively. They were 27 68 years old, averagely $(42.6 \pm 6.4)$ years. Their body weight was $56 \sim 79 \mathrm{~kg}$, $(65.2 \pm 8.5) \mathrm{kg}$ on average. Inclusion criteria: a. meeting a criterion of America Society of Anesthesiologist (ASA) grading I II; b. absence of serious heart and lung disease, coagulation disorders, liver and kidney dysfunction; c. absence of chronic disease and anaesthetic allergic history; d. unused anaphylactic drugs in recent 3 years. The patients were randomly divided into three groups, 19 cases in each group. Trial group I: There were 8 males and 9 females. They were $(43.2 \pm 6.2)$ years old, and the body weight was $(64.9 \pm 8.1) \mathrm{kg}$. Operation time was $(125.0 \pm 27.5) \mathrm{min}$. Trial group II: There were 9 males and 10 females. They were at the age of $(42.1 \pm 6.6)$, and the body weight was $(65.5 \pm 8.7) \mathrm{kg}$. Operation time was (128.7 \pm 28.2$) \mathrm{min}$. Control group: The males and females were 7 and 12 cases, respectively. They were at the age of (42.3 \pm 6.4$)$. The body weight and operation time were $(65.9 \pm 8.0) \mathrm{kg}$ and $(126.3 \pm 27.6) \mathrm{min}$, respectively. There was no statistical significance among three groups by comparison to the gender, age, body weight and operation time $(P>0.05)$, with better comparability.

\section{Methods}

Control group was intravenously injected $4 \mathrm{~mL}$ of $0.9 \%$ sodium chloride injection before anesthesia induction; Trial group I was intravenously injected $40 \mathrm{mg}$ of parecoxib sodium (Pfizer Pharmaceuticals Limited) 20 min before anesthesia induction; Based on trial group I, trial group II was injected $40 \mathrm{mg}$ of parecoxib sodium again $12 \mathrm{~h}$ after surgery.
Before, 12 and $24 \mathrm{~h}$ after surgery, $5 \mathrm{~mL}$ of venous blood from fasting patients was drawn in three groups to detect the levels of plasma norepinephrine (NE), cortisol (Cor) and blood glucose. Plasma NE and Cor contents were detected respectively using high performance liquid chromatography electrochemical detector (HPLC-ECD) and radioimmunoassay. HPviridia24c multi-function monitor was applied to continuously monitor the heart rate (HR), systolic pressure (SBP), diastolic pressure (DBP) and sphygmous oxygen saturation (SpO2), and the level of each index was recorded before surgery, at the end of surgery as well as 12, 24 and $48 \mathrm{~h}$ after surgery. Meanwhile, visual analogue scale (VAS) scores were used for evaluating the analgesic effect 4, 8, 12 and $24 \mathrm{~h}$ after surgery.

\section{Observational indexes}

The levels of plasma NE, Cor and blood glucose before, 12 and $24 \mathrm{~h}$ after surgery and changes of haemodynamics, including $\mathrm{HR}, \mathrm{SBP}, \mathrm{DBP}$ and $\mathrm{SpO}_{2}$, before surgery, at the end of surgery and 12, 24 and 48 $\mathrm{h}$ after surgery were compared in three groups. Besides, VAS scores were recorded 4, 8, 12 and $24 \mathrm{~h}$ after surgery.

\section{Evaluation criteria}

Scoring criteria for VAS analgesia: 0 point (Painless); 1 4 points (mild pain); 5 6 points (moderate pain); 7 9 points (severe pain) and 10 points (unbearable pain).

\section{Statistical data analysis}

SPSS 15.0 statistical software was used to analyze the data statistically. Enumeration data were compared by $\mathrm{x}^{2}$ test, expressed with percentages; Measurement data were compared by $t$ test, expressed with mean \pm standard deviation $(\bar{x} \pm s) . P<0.05$ was considered to be statistically significant.

\section{Results}

Comparison on the levels of plasma NE, Cor and blood glucose in three groups at different time points

There was no statistical significance among three groups by comparison to the levels of plasma NE, Cor and blood glucose before surgery $(P>0.05) .12$ and $24 \mathrm{~h}$ after surgery, the levels of plasma NE and Cor in three groups went up dramatically $(P<0.05$ or $P<0.01)$; The levels of plasma NE and Cor in trial groups I and II were evidently

Table 1. Comparison on the Levels of Plasma NE, Cor and Blood Glucose in three Groups at Different Time Points $(\bar{x} \pm s)$

\begin{tabular}{|c|c|c|c|c|}
\hline Groups & Time & $\mathrm{NE}(\mathrm{mg} / \mathrm{L})$ & Cor $(\mathrm{mg} / \mathrm{L})$ & Blood glucose $(\mathrm{mmol} / \mathrm{L})$ \\
\hline \multirow[t]{3}{*}{ Control group $(n=19)$} & Before surgery & $225.92 \pm 134.86$ & $210.39 \pm 38.27$ & $5.18 \pm 0.45$ \\
\hline & $12 \mathrm{~h}$ after surgery & $386.05 \pm 119.98 * *$ & $262.30 \pm 37.09 * *$ & $5.43 \pm 0.42$ \\
\hline & $24 \mathrm{~h}$ after surgery & $392.64 \pm 118.83 * *$ & $269.48 \pm 35.40 * *$ & $5.48 \pm 0.41$ \\
\hline \multirow[t]{3}{*}{ Trial group I $(n=19)$} & Before surgery & $220.97 \pm 122.86$ & $207.26 \pm 37.07$ & $5.09 \pm 0.49$ \\
\hline & $12 \mathrm{~h}$ after surgery & $303.01 \pm 123.91 * \#$ & $236.91 \pm 38.09 * \#$ & $5.32 \pm 0.65$ \\
\hline & $24 \mathrm{~h}$ after surgery & $313.22 \pm 114.60 * \#$ & $244.08 \pm 34.39 * \#$ & $5.40 \pm 0.48$ \\
\hline \multirow[t]{3}{*}{ Trial group II $(n=19)$} & Before surgery & $217.03 \pm 124.47$ & $206.77 \pm 36.98$ & $5.10 \pm 0.46$ \\
\hline & $12 \mathrm{~h}$ after surgery & $298.06 \pm 120.89 * \#$ & $231.78 \pm 34.46 * \#$ & $5.28 \pm 0.45$ \\
\hline & $24 \mathrm{~h}$ after surgery & $302.45 \pm 115.39 * \#$ & $234.79 \pm 33.41 * \# \#$ & $5.36 \pm 0.39$ \\
\hline
\end{tabular}


Preemptive Analgesia with Parecoxib Sodium Reduces Plasma Stress Hormones in Surgical Patients with Thyroid Carcinoma Table 2. Comparison on Hemodynamic Levels in three Groups at Different Time Points $(\bar{x} \pm s, n=19)$

\begin{tabular}{|c|c|c|c|c|c|c|}
\hline Indexes & Groups & $\begin{array}{l}\text { Before } \\
\text { surgery }\end{array}$ & $\begin{array}{l}\text { At the end of } \\
\text { surgery }\end{array}$ & $\begin{array}{c}12 \mathrm{~h} \text { after } \\
\text { surgery }\end{array}$ & $\begin{array}{c}24 \mathrm{~h} \text { after } \\
\text { surgery }\end{array}$ & $\begin{array}{c}48 \mathrm{~h} \text { after } \\
\text { surgery }\end{array}$ \\
\hline \multirow[t]{3}{*}{ HR (time/point) } & Control group & $65.08 \pm 4.59$ & $78.19 \pm 8.68 * *$ & $78.91 \pm 5.86 * *$ & $69.03 \pm 5.01 *$ & $69.77 \pm 8.48 *$ \\
\hline & Trial group I & $64.32 \pm 5.59$ & $69.01 \pm 8.99 \# \#$ & $73.71 \pm 7.18 * * \#$ & $66.82 \pm 4.04$ & $67.18 \pm 7.73$ \\
\hline & Trial group II & $65.25 \pm 5.28$ & $67.73 \pm 5.69 \# \#$ & $70.68 \pm 7.02 * \# \#$ & $64.78 \pm 6.03 \#$ & $66.08 \pm 7.79$ \\
\hline \multirow[t]{3}{*}{ SBP (mmHg) } & Control group & $130.48 \pm 4.89$ & $138.02 \pm 6.81 * *$ & $140.72 \pm 6.03 * *$ & $133.91 \pm 6.12$ & $129.89 \pm 7.81$ \\
\hline & Trial group I & $129.19 \pm 4.79$ & $131.74 \pm 7.48 \#$ & $136.13 \pm 6.80 * * \#$ & $130.03 \pm 7.06$ & $127.79 \pm 6.92$ \\
\hline & Trial group II & $129.69 \pm 5.01$ & $130.08 \pm 7.58 \# \#$ & $134.49 \pm 6.68 * \# \#$ & $128.88 \pm 6.86 \#$ & $126.89 \pm 6.12$ \\
\hline \multirow[t]{3}{*}{ DBP (mmHg) } & Control group & $72.63 \pm 6.30$ & $80.49 \pm 6.08 * *$ & $75.03 \pm 6.21$ & $70.79 \pm 7.02$ & $68.61 \pm 6.12$ \\
\hline & Trial group I & $71.73 \pm 6.03$ & $75.14 \pm 6.29 \#$ & $70.18 \pm 6.79 \#$ & $68.91 \pm 6.92$ & $68.67 \pm 8.11$ \\
\hline & Trial group II & $72.08 \pm 5.14$ & $75.09 \pm 6.28 \#$ & $69.09 \pm 5.50 \#$ & $69.11 \pm 7.10$ & $68.12 \pm 6.89$ \\
\hline \multirow[t]{3}{*}{$\mathrm{SpO}_{2}(\%)$} & Control group & $98.02 \pm 0.43$ & $98.07 \pm 0.79$ & $97.89 \pm 0.99$ & $97.78 \pm 0.81$ & $97.98 \pm 0.79$ \\
\hline & Trial group I & $98.09 \pm 0.51$ & $97.97 \pm 0.69$ & $97.62 \pm 1.02$ & $98.09 \pm 0.82$ & $98.37 \pm 0.61$ \\
\hline & Trial group II & $97.99 \pm 0.47$ & $98.78 \pm 0.72$ & $97.97 \pm 0.87$ & $97.69 \pm 0.91$ & $98.06 \pm 0.52$ \\
\hline
\end{tabular}

Compared with surgery before, $* \mathrm{P}<0.05$, $* * \mathrm{P}<0.01$; Compared with control group, \#P<0.05, \#\#P<0.01

Table 3. Comparison on VAS Scores in three Groups at Different Time Points $(\bar{x} \pm s)$

\begin{tabular}{|c|c|c|c|c|c|}
\hline Groups & $\mathrm{n}$ & $4 \mathrm{~h}$ after surgery & $8 \mathrm{~h}$ after surgery & $12 \mathrm{~h}$ after surgery & $24 \mathrm{~h}$ after surgery \\
\hline Control group & 19 & $6.1 \pm 1.0$ & $5.8 \pm 0.7$ & $5.5 \pm 0.6$ & $5.2 \pm 0.4$ \\
\hline Trial group I & 19 & $5.2 \pm 0.9 * *$ & $4.4 \pm 0.8 * *$ & $3.9 \pm 0.7 * *$ & $3.6 \pm 0.8 * *$ \\
\hline Trial group II & 19 & $4.4 \pm 0.8 * * \# \#$ & $3.8 \pm 0.7 * * \#$ & $3.4 \pm 0.6 * * \#$ & $3.1 \pm 0.5 * * \#$ \\
\hline
\end{tabular}

Compared with control group, ${ }^{* *} P<0.01$; Compared with trial group I, ${ }^{\#} P<0.05,{ }^{\# \#} P<0.01$

lower than in control group $(P<0.05$ or $\mathrm{P}<0.01)$, and those in trial group II slightly lower than in trial group I, but no significant difference was indicated $(P>0.05)$. There was also no statistical significance among three groups regarding to the levels of blood glucose before, 12 and $24 \mathrm{~h}$ after surgery $(P>0.05)$ (Table 1$)$.

Comparison on hemodynamic levels in three groups at different time points

There was no statistical significance among three groups by comparison to the HR, SBP, DBP and $\mathrm{SpO}_{2}$ level before surgery $(P>0.05)$. The HR in control group increased dramatically at the end of surgery, 12, 24 and $48 \mathrm{~h}$ after surgery; $12 \mathrm{~h}$ after surgery, the HR and SBP in trial groups I and II increased obviously by comparison to surgery before $(P<0.05$ or $P<0.01)$, but gradually returned to the preoperative level. The HR, SBP and DBP in trial groups I and II at the end of surgery and $12 \mathrm{~h}$ after surgery were all lower than in control group, and significant differences were presented $(P<0.05$ or $P<0.01)$. There was also no statistical significance between trial groups I and II with regard to the level of each index at the end of surgery, 12, 24 and $48 \mathrm{~h}$ after surgery $(P>0.05)$ (Table 2$)$.

Comparison on VAS scores in three groups at different time points

VAS scores in each group were decreased by varying degrees $4 \mathrm{~h}$ after surgery. 4, 8, 12 and $24 \mathrm{~h}$ after surgery, VAS scores in trial groups I and II were markedly lower than in control group $(P<0.05$ or $P<0.01)$, in which the scores in trial group II were the lowest (Table 3 ).

\section{Discussion}

As the most common malignant tumor in endocrine system, thyroid carcinoma approximately accounts for $92 \%$, in which the most commonly-encountered papillary thyroid carcinoma (PTC) can occur at any age (Haselkorn et al., 2000). A number of research results displayed that the growth rate of PTC was the fast, especially that of tiny papillary carcinoma (Ranjbari et al., 2013; Sungwalee et al., 2013; Oakley et al., 2014). The etiology of thyroid carcinoma is still unclear at present, considering that it may be related to thyroid autoimmune disease, familial inheritance, estrogen level, external exposure of radioactive rays and improper intake of iodine (Xhaard et al., 2014).

The comprehensive treatment dominated by surgery is still an important way to treat thyroid carcinoma, and positive surgery can improve the patient's longterm survival rate. Nevertheless, as a sort of noxious stimulation, the surgery can be involved in activation and modulation of nociceptors through induction of peripheral tissue to release and generate various chemokines and cytokines, consequently resulting in negative effects on the body. Surgical trauma can make the body generate stress responses conspicuously, induce increase of COX-2 expression and prostaglandin E release, activate prostaglandin E receptors of peripheral nociceptors and increase stimulating sensitivity so as to cause hyperpathia (Rasmussen et al., 2002). Furthermore, the sensitization of central nervous system during surgery can also prolong and diffuse postoperative pain. Stress responses are a key factor that affects postoperative prognosis, and severe ones can make metabolism and homeostasis in disorder. Nowadays, a variety of therapeutic measures have been clinically adopted to regulate the stress response in perioperative period, primarily including application of minimally invasive surgery, development and perfection of anesthetic techniques as well as development and application of anesthetics and non-anesthetics. Good postoperative analgesia could inhibit the body stress response, promote wound healing, decrease the incidence of complications, relieve pain and was conductive to 


\section{Lian-Dong Wang et al}

postoperative rehabilitation (Ezhevskaya et al., 2013; Das et al., 2014).

Taken prior to the action of noxious stimulation on the body, preemptive analgesia can prevent peripheral and central nervous systems from sensitization. Parecoxib sodium, a highly-selective COX-2 inhibitor for injection, has dual analgesic effects on peripheral and central nervous systems. By inhibiting the transformation of arachidonic acid to prostaglandin selectively, it can lessen the synthesis of prostaglandin and inhibit hyperpathia to exert postoperative analgesic and antiinflammatory effects (Talley et al., 2000; Kyriakidis et al., 2011). Parecoxib sodium itself has no functions of inhibiting COX-1 and COX-2, but can be hydrolyzed into valdecoxib and propionic acid rapidly by enzymes after intravenous injection. However, valdecoxib pertains to a highly-selective COX-2 inhibitor. Its safety is higher than conventional non-steroidal anti-inflammatory drugs in gastrointestinal tract and impact on platelets is milder (Lloyd et al., 2009). The research results in this study displayed that 12 and $24 \mathrm{~h}$ after surgery, the levels of plasma NE and Cor in three groups went up dramatically; The levels of plasma NE and Cor in trial groups I and II were evidently lower than in control group, and those in trial group II slightly lower than in trial group I, indicating that more serious stress responses appeared in control group that showed larger fluctuation of plasma stress hormones, then in trial group I, and the trial group II had the smallest stress responses. It is probably because parecoxib sodium decreases the sensitivity of sensory nerve fibers to noxious stimulation to perform the functions of analgesia and anti-inflammation, thereby inhibiting the secretion of stress hormones like NE and Cor, via inhibition of COX-2 expression to affect the metabolism of arachidonic acid and synthesis of prostaglandin. $12 \mathrm{~h}$ after surgery, the HR and SBP in trial groups I and II increased obviously by comparison to surgery before, but gradually returned to the preoperative level. HR, SBP and DBP in trial groups $\mathrm{I}$ and II at the end of surgery and $12 \mathrm{~h}$ after surgery were all lower than in control group, showing that parecoxib sodium had a little influence on haemodynamics at the end of surgery and $12 \mathrm{~h}$ after surgery, but this influence gradually disappeared as time went on. 4, 8, 12 and 24 $\mathrm{h}$ after surgery, VAS scores in trial groups I and II were markedly lower than in control group, in which the scores in trial group II were the lowest, demonstrating that injection of parecoxib sodium before anesthesia and $12 \mathrm{~h}$ after surgery could effectively enhance the effect of preemptive analgesia.

To sum up, combined application of parecoxib sodium for preemptive analgesia before anesthesia and after surgery can effectively inhibit the levels of plasma stress hormones and improve analgesic effect in surgical patients with thyroid carcinoma, and without conspicuous impact on haemodynamics.

\section{References}

Cramer JD, Fu P, Harth KC, et al (2010). Analysis of the rising incidence of thyroid cancer using the Surveillance, Epidemiology and End Results nationalcancer data registry.
Surgery, 148, 1147-52.

Das J, Kumar S, Khanna S, et al (2014). Are we causing the recurrence-impact of perioperative period on long-term cancer prognosis: Review of current evidence and practice. $J$ Anaesthesiol Clin Pharmacol, 30, 153-9.

Ezhevskaya AA, Mlyavykh SG, Anderson DG (2013). Effects of continuous epidural anesthesia and postoperative epidural analgesia on pain management and stressresponse in patients undergoing major spinal surgery. Spine (Phila Pa 1976), 38, 1324-30.

Haselkorn T, Bernstein L, Preston-Martin S, et al (2000). Descriptive epidemiology of thyroid cancer in Los Angeles County, 1972-1995. Cancer Causes Control, 11, 163-70.

Kyriakidis AV, Perysinakis I, Alexandris I, et al (2011). Parecoxib sodium in the treatment of postoperative pain after Lichtenstein tension-free mesh inguinal hernia repair. Hernia, 15, 59-64.

Lloyd R, Derry S, Moore RA, et al (2009). Intravenous or intramuscular parecoxib for acute postoperative pain in adults. Cochrane Database Syst Rev, 15, 4771.

Oakley GM, Curtin K, Layfield L, et al (2014). Increased melanoma risk in individuals with papillary thyroid carcinoma. JAMA Otolaryngol Head Neck Surg, 140, 423-7.

Persec J, Persec Z, Bukovic D, et al (2007). Effects of clonidine peempt analgesia on acute postoperative pain in abdominal surgery. Coll Antropol, 31, 1071-5.

Ranjbari N, Almasi S, Mohammadi-Asl J, et al (2013). BRAF mutations in Iranian patients with papillary thyroid carcinoma. Asian Pac J Cancer Prev, 14, 2521-3.

Rasmussen GL, Steckner K, Hogue C, et al (2002). Intravenous parecoxib sodium foracute pain after orthopedic knee surgery. Am J Orthop (Belle Mead NJ), 31, 336-43.

Sandhu T, Paiboonworachat S, Ko-iam W (2011). Effects of preemptive analgesia in laparoscopic cholecystectomy: a double-blind randomized controlled trial. Surg Endosc, 25, 23-7.

Sungwalee W, Vatanasapt P, Kamsa-Ard S, et al (2013). Reproductive risk factors for thyroid cancer: a prospective cohort study in Khon Kaen, Thailand. Asian Pac J Cancer Prev, 14, 5153-5.

Talley JJ, Bertenshaw SR, Brown DL, et al (2000). N-[[(5-methyl3-phenylisoxazol-4-yl)-phenyl]sulfonyl] propanamide, sodium salt, parecoxib sodium: A potent and selective inhibitor of COX-2 for parenteral administration. $J$ Med Chem, 43, 1661-3.

Wei W, Zhao T, Li Y (2013). Efficacy and safety of parecoxib sodium for acute postoperative pain: A meta-analysis. Exp Ther Med, 6, 525-31.

Xhaard C, Ren Y, Clero E, et al (2014). Differentiated thyroid carcinoma risk factors in French Polynesia. Asian Pac $J$ Cancer Prev, 15, 2675-80. 